\title{
Characteristics and treatment regimens across ERS SHARP severe asthma registries
}

\author{
Job J.M.H. van Bragt (10 ${ }^{1}$, Ian M. Adcock ${ }^{2}$, Elisabeth H.D. Bel ${ }^{1}$, \\ Gert-Jan Braunstahl ${ }^{3}$, Anneke ten Brinke ${ }^{4}$, John Busby ${ }^{5}$, Giorgio W. Canonica ${ }^{6}$, \\ Hui $\mathrm{Cao}^{7}$, Kian Fan Chung ${ }^{8}$, Zsuzsanna Csoma ${ }^{9}$, Barbro Dahlén ${ }^{10}$, \\ Elizabeth Davin ${ }^{11}$, Susanne Hansen ${ }^{12}$, Enrico Heffler ${ }^{6}$, Ildiko Horvath ${ }^{9}$, \\ Stephanie Korn ${ }^{13}$, Maxim Kots ${ }^{14}$, Piotr Kuna ${ }^{15}$, Namhee Kwon ${ }^{16}$, \\ Renaud Louis ${ }^{17}$, Vicente Plaza (18 ${ }^{18}$, Celeste Porsbjerg ${ }^{19}$, David Ramos-Barbon ${ }^{18}$, \\ Levi B. Richards (101, Sabina Škrgat (1020 ${ }^{20}$, Jacob K. Sont ${ }^{21}$, Susanne J.H. Vijverberg (1) ${ }^{1}$ \\ Els J.M. Weersink ${ }^{1}$, Valentyna Yasinska ${ }^{10}$, Scott S. Wagers ${ }^{22}$, Ratko Djukanovic ${ }^{23}$ \\ and Anke H. Maitland-van der Zee ${ }^{1}$ on behalf of the SHARP Clinical Research \\ Collaboration ${ }^{24}$
}

@ERSpublications

The severe asthma population in Europe is heterogeneous and differs in clinical characteristics and treatment. Harmonisation across registries and guidelines is needed and requires collection of same data across cohorts to enable future research in SHARP. http://bit.ly/2krxHPf

Cite this article as: van Bragt JJMH, Adcock IM, Bel EHD, et al. Characteristics and treatment regimens across ERS SHARP severe asthma registries. Eur Respir J 2020; 55: 1901163 [https://doi.org/10.1183/ 13993003.01163-2019].

ABSTRACT Little is known about the characteristics and treatments of patients with severe asthma across Europe, but both are likely to vary. This is the first study in the European Respiratory Society Severe Heterogeneous Asthma Research collaboration, Patient-centred (SHARP) Clinical Research Collaboration and it is designed to explore these variations. Therefore, we aimed to compare characteristics of patients in European severe asthma registries and treatments before starting biologicals.

This was a cross-sectional retrospective analysis of aggregated data from 11 national severe asthma registries that joined SHARP with established patient databases.

Analysis of data from 3236 patients showed many differences in characteristics and lifestyle factors. Current smokers ranged from 0\% (Poland and Sweden) to 9.5\% (Belgium), mean body mass index ranged from 26.2 (Italy) to $30.6 \mathrm{~kg} \cdot \mathrm{m}^{-2}$ (the UK) and the largest difference in mean pre-bronchodilator forced expiratory volume in $1 \mathrm{~s} \%$ predicted was $20.9 \%$ (the Netherlands versus Hungary). Before starting biologicals patients were treated differently between countries: mean inhaled corticosteroid dose ranged from 700 to $1335 \mu \mathrm{g} \cdot \mathrm{day}^{-1}$ between those from Slovenia versus Poland when starting anti-interleukin (IL)5 antibody and from 772 to $1344 \mu \mathrm{g} \cdot \mathrm{day}^{-1}$ in those starting anti-IgE (Slovenia versus Spain). Maintenance oral corticosteroid use ranged from 21.0\% (Belgium) to 63.0\% (Sweden) and from 9.1\% (Denmark) to $56.1 \%$ (the UK) in patients starting anti-IL-5 and anti-IgE, respectively.

The severe asthmatic population in Europe is heterogeneous and differs in both clinical characteristics and treatment, often appearing not to comply with the current European Respiratory Society/American Thoracic Society guidelines definition of severe asthma. Treatment regimens before starting biologicals were different from inclusion criteria in clinical trials and varied between countries.

This article has supplementary material available from erj.ersjournals.com

Received: 14 June 2019 | Accepted after revision: 18 Sept 2019

Copyright OERS 2020 


\section{Introduction}

The European Respiratory Society (ERS) Severe Heterogeneous Asthma Research collaboration, Patient-centred (SHARP) Clinical Research Collaboration (CRC) was set up in 2018 to harmonise severe asthma management across Europe and unravel underlying heterogeneity in a patient-centred way [1]. The current project involves the first structured assessment and comparison of national severe asthma registries that are part of SHARP to discover strengths/weaknesses in those registries and to evaluate severe asthma and its treatment across Europe.

Significant progress has been made in the field of severe asthma since the turn of the millennium [2]. In particular, the introduction of novel biologicals for patients with severe asthma has provided new effective options for medical treatment, beginning with the anti-IgE monoclonal antibody, omalizumab, and more recently anti-interleukin (IL)-5 antibodies, mepolizumab, reslizumab and benralizumab. The use of these biologicals is often restricted to patients who fulfil the definition of severe asthma according to ERS/ American Thoracic Society (ATS) guidelines in whom all potential aggravating factors have been eliminated and pre-specified criteria fulfilled, such as high-dose inhaled corticosteroid (ICS)/long-acting $\beta$-agonist (LABA) treatment, multiple exacerbations in the previous year and/or chronic use of oral corticosteroids (OCSs) [3].

The most recent Global Initiative for Asthma difficult-to-treat and severe asthma guide introduces a new approach to the management of asthma [4], better reflecting the recommendations of The Lancet Commission on asthma [5,6] that highlighted the need for a multidimensional assessment and the introduction of treatable mechanisms in asthma management. However, the definition of severe asthma is still not unambiguous and it has been suggested that an improved definition, including risk assessment and a better reflection of clinical reality, should be established. Asthma death is arguably the most severe outcome, but most deaths occur in patients with nonsevere asthma with low levels of treatment [7]. Many disease aggravating factors, both patient related (e.g. psychological factors, comorbidities) and environmental (airborne allergens and air pollution), socioeconomic factors (housing and health insurance) and healthcare accessibility factors are difficult or impossible to eliminate. Furthermore, the expertise of the treating physician and the facilities of the treatment centre are likely to influence levels of asthma control. Finally, the choices of treatment (including starting biologicals) are effected by differences in healthcare systems, reimbursement policies and accessibility to medication.

In the present study, we explored the prevalence as well as the characteristics of patients with "severe asthma" reported by physicians, which are likely to differ depending on the region, climate, healthcare system and expertise of the treatment team. Furthermore, we explored the severity of disease (using the treatment of the patient and biomarkers as a proxy) of severe asthma patients before starting biologicals, also expecting differences between European countries. We compared the characteristics of those patients who started with high-cost therapies (biologicals, bronchial thermoplasty and high-altitude revalidation) within these registries and stratified the patient cohort for the two most commonly used groups of biologicals (anti-IL-5 and anti-IgE). This study showed the need for harmonisation across registries and

Affiliations: ${ }^{1}$ Dept of Respiratory Medicine, Amsterdam UMC, University of Amsterdam, Amsterdam, The Netherlands. ${ }^{2}$ Molecular Cell Biology Group, National Heart and Lung Institute, Imperial College London, London, UK. ${ }^{3}$ Franciscus Gasthuis and Vlietland, Rotterdam, The Netherlands. ${ }^{4}$ Medical Centre Leeuwarden, Leeuwarden, The Netherlands. ${ }^{5}$ Centre for Public Health, School of Medicine, Dentistry and Biomedical Sciences, Queen's University Belfast, Belfast, UK. 'Personalised Medicine Clinic, Asthma and Allergy, Humanitas Clinical and Research Centre, Humanitas University, Rozzano and SANI-Severe Asthma Network Italy, Milan, Italy. ${ }^{7}$ Novartis Pharmaceuticals, East Hanover, NJ, USA. ${ }^{8}$ Experimental Studies Medicine, National Heart and Lung Institute, Imperial College London, London, UK. ${ }^{9}$ National Koranyi Institute of Pulmonology, Budapest, Hungary. ${ }^{10}$ Division of Respiratory Medicine and Allergy, Dept of Medicine, Karolinska University Hospital, Huddinge, Sweden. ${ }^{11}$ European Lung Foundation, Sheffield, UK. ${ }^{12}$ Center for Clinical Research and Disease Prevention, Bispebjerg and Frederiksberg Hospital, Copenhagen, Denmark. ${ }^{13}$ Universitätsmedizin Mainz, Mainz, Germany. ${ }^{14}$ Global Clinical Development, Chiesi Farmaceutici, Parma, Italy. ${ }^{15}$ Dept of Internal Medicine, Asthma and Allergy, Medical University of Lodz, Lodz, Poland. ${ }^{16}$ Respiratory Medical Franchise, GSK, Brentford, UK. ${ }^{17}$ Dept of Pulmonary Medicine, Centre Hospitalier Universitaire, GIGA-I3 Research Group, Liege University, Liege, Belgium. ${ }^{18}$ Respiratory Medicine Dept and Biomedical Research Institute, Hospital de la Santa Creu i Sant Pau, Universitat Autònoma de Barcelona, Barcelona, Spain. ${ }^{19}$ Respiratory Research Unit, Dept of Respiratory Medicine, Bispebjerg University Hospital, Copenhagen, Denmark. ${ }^{20}$ University Clinic of Respiratory and Allergic Diseases, Golnik, Slovenia. ${ }^{21}$ Dept of Biomedical Data Sciences, Section Medical Decision Making, Leiden University Medical Center, Leiden, The Netherlands. ${ }^{22}$ BioSciConsulting, Maasmechelen, Belgium. ${ }^{23}$ NIHR Southampton Respiratory Biomedical Research Unit, Faculty of Medicine, University of Southampton, Southampton, UK. ${ }^{24}$ Members of the SHARP Clinical Research Collaboration are listed in the Acknowledgements section.

Correspondence: Job J.M.H. van Bragt, Dept of Respiratory Medicine, Amsterdam UMC, F5-260, Meibergdreef 9, 1105 AZ Amsterdam, The Netherlands. E-mail: j.j.vanbragtdamsterdamumc.nl 
FIGURE 1 Participating countries in the current study in the European Respiratory Society Severe Heterogeneous Asthma Research collaboration, Patient-centred

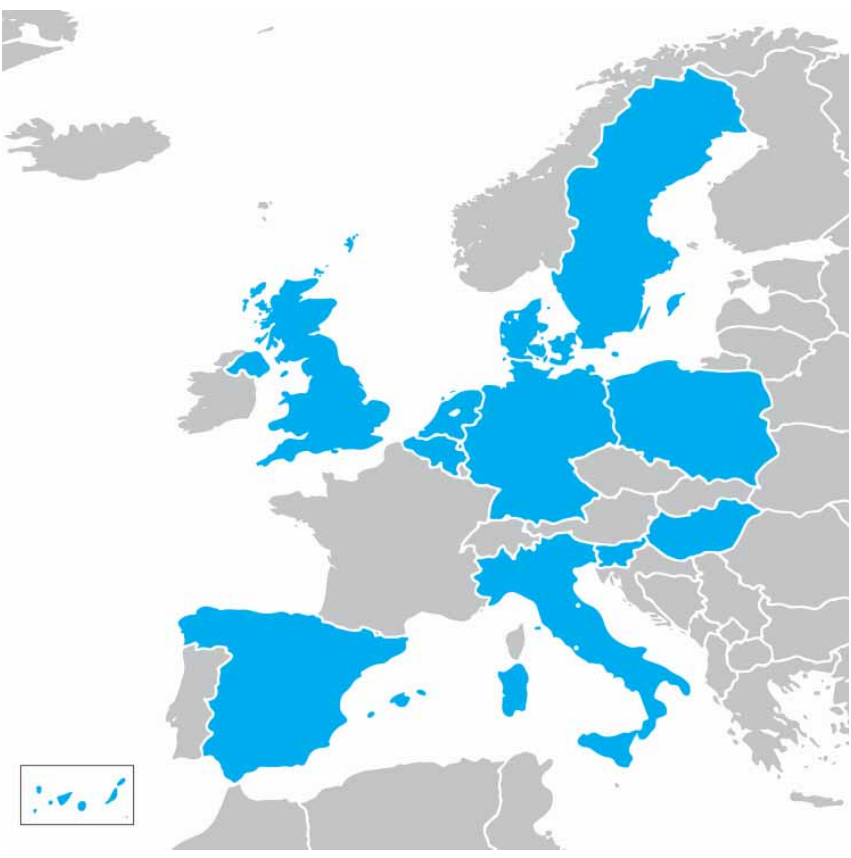
(SHARP).

guidelines, and the requirement to collect the same set of minimal clinical data across cohorts which will enable better coordination of treatment efforts using biologicals across Europe.

\section{Materials and methods \\ Study subjects}

Data from 11 different European national registries for severe asthma were eligible for inclusion in the analysis (figure 1). There were no general inclusion criteria provided for the patients in these registries, so these differed between registries (figure 2). Most European registries included patients who fulfilled the severe asthma criteria according to the joint ERS/ATS guidelines [3], but in some cases national asthma guidelines were used or all patients who attended specialist asthma centres were qualified for inclusion. Four registries focused enrolment into registries of patients that were either in the process of being considered for or were starting treatment with biologicals. One registry selected only patients with a smoking history $<5$ pack-years and one registry included all patients who attended specialist referral centres for severe asthma.

\section{Study design}

This study was a cross-sectional, retrospective analysis of aggregated registry data. In view of the restrictions imposed on data confidentiality before and, in some cases, after imposition in the European Union of the General Data Protection Regulation, data were received from individual national registries in aggregate form composed of counts (percentages), mean with standard deviation and median (interquartile range (IQR)).
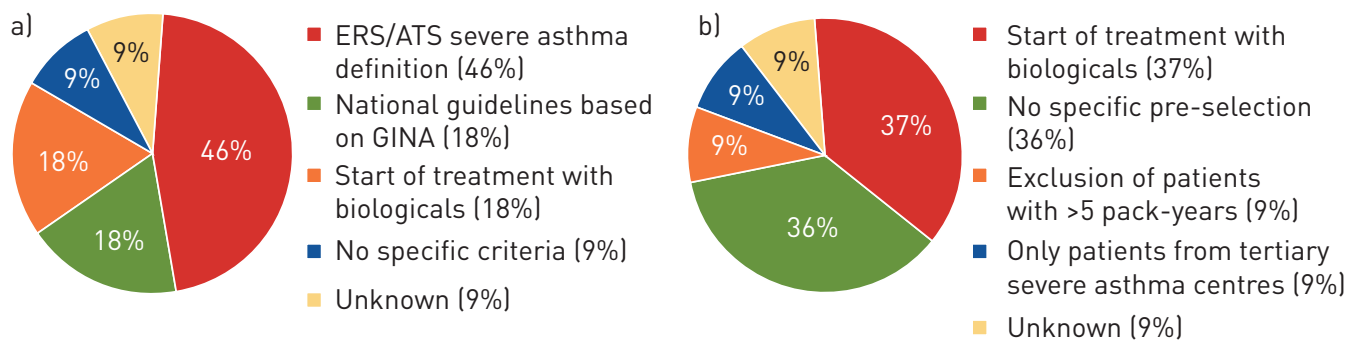

FIGURE 2 a) Inclusion criteria and b) criteria for pre-selection of patients in the different registries. ERS: European Respiratory Society; ATS: American Thoracic Society; GINA: Global Initiative for Asthma. 


\section{Analysis}

A descriptive comparison was performed for the clinical characteristics in the different registries. To describe differences in treatment regimens and biomarkers, comparisons were made prior to starting high-cost therapies. For those patients who had already discontinued these therapies, and where information before start was not available, data from $>6$ months after stopping high-cost therapies were used. High-cost therapies were defined as treatments with biologicals, bronchial thermoplasty or high-altitude treatment.

\section{Results}

Data from a total of 3236 patients classified as having severe asthma by the 11 different European registries were used for the analysis. The registries varied in size ( $n=27$ in Sweden to $n=765$ in the UK). The characteristics of the different populations are shown in table 1 (see supplementary material for further details). None of the participating registries collected the full set of variables requested. Data that were not collected or not available in a registry are indicated in the various tables.

\section{Differences in baseline clinical characteristics}

The mean age of patients ranged from 44.4 (Germany) to 58.3 years (Hungary). The percentage of males ranged from $30.0 \%$ (Slovenia) to $51.9 \%$ (Sweden). Current smokers ranged from $0 \%$ (Poland and Sweden) to $9.5 \%$ (Belgium). The percentage of ex-smokers ranged from $10.8 \%$ (Hungary) to $41.3 \%$ (the Netherlands); in Hungary, only patients with a smoking history $<5$ pack-years were enrolled into the registry. Over half of the registries predominantly included patients with adult-onset severe asthma (the Netherlands, the UK, Hungary, Sweden, Denmark and Slovenia), while four registries consisted mainly of patients with childhood-onset asthma (Belgium, Italy, Poland and Germany). Based on mean forced expiratory volume in $1 \mathrm{~s}\left(\mathrm{FEV}_{1}\right)$ and forced vital capacity (FVC) (\% pred), patients in the Dutch registry had the best lung function, while those in the Hungarian registry had the worst lung function $\left(\mathrm{FEV}_{1}\right.$ $76.9 \%$ versus $56.0 \%$ and FVC $98.3 \%$ versus $76.6 \%$ ). Median blood eosinophil levels ranged from $0.230 \times 10^{9}$ (the Netherlands) to $0.800 \times 10^{9}$ cells $\cdot \mathrm{L}^{-1}$ (Sweden), median serum total IgE ranged from 144 (the Netherlands) to $275 \mathrm{IU} \cdot \mathrm{mL}^{-1}$ (Sweden) and median exhaled nitric oxide fraction ( $\left.F_{\mathrm{ENO}}\right)$ ranged from 25 (Belgium) to $66 \mathrm{ppb}$ (Slovenia). Between 54.6\% (Italy) and 100\% (Hungary and Sweden) of the patients were uncontrolled as judged from patient-reported questionnaire scores (Asthma Control Questionnaire or Asthma Control Test).

Most registries enrolled patients being treated in a tertiary care centre; however, a small group of patients was included in primary care (Spain) and four registries (Spain, the Netherlands, Belgium and Hungary) included up to $33.8 \%$ in secondary care hospitals. In most registries $>90 \%$ of the patients were treated according to GINA step 4 or 5 guidelines [7]; in Denmark this was $77.6 \%$ (evaluated before patients started biologicals) and in six registries (Hungary, Poland, Sweden, Germany, Italy and Slovenia) 100\% of patients were at GINA step 4 or 5 . The percentage of patients on biologicals ranged from $0 \%$ (Sweden) to $71.0 \%$ (Poland). The most frequently given biological in seven registries was anti-IgE (Belgium, Spain, Hungary, Poland, Germany, Italy and Slovenia) and in three registries it was anti-IL-5 (the Netherlands, the UK and Denmark). The registries in the Netherlands, Belgium and Slovenia enrolled patients who had undergone bronchial thermoplasty, and the registry in the Netherlands also included patients who had received high-altitude treatment (14\%). The mean \pm SD ICS dose (fluticasone equivalent) ranged from $491 \pm 163$ (Slovenia) to $1225 \pm 445 \mu \mathrm{g} \cdot \mathrm{day}^{-1}$ (Spain). The maintenance OCS median dose ranged from 7.5

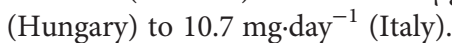

\section{Treatment regimens of patients starting high-cost therapies}

Table 2 shows medication data of 1960 patients included in the registries prior to starting or $>6$ months after stopping high-cost therapies. In six registries (Belgium, Spain, Hungary, Sweden, Slovenia and Denmark), most patients were not treated according to GINA step 5 treatment. Short-acting $\beta$-agonists (SABAs) were the most used reliever medications in all but one registry (Spain) where the most frequent relievers were short-acting muscarinic antagonists (SAMAs). SABA and/or SAMA use ranged from $4.0 \%$ (Italy) to $100 \%$ (Hungary, Poland and Sweden). LABA use ranged from $80.3 \%$ (Poland) to $100 \%$ (Italy, Hungary and Sweden). Long-acting muscarinic antagonists (LAMAs) were used in all countries and ranged from $14.0 \%$ (Hungary) to $56.4 \%$ (the UK). In seven registries (Spain, Hungary, Poland, Sweden, Italy, Slovenia and the UK) all patients were on ICS, while in the Netherlands, Belgium and Denmark this was $99.3 \%, 97.4 \%$ and $93.9 \%$, respectively. Mean \pm SD fluticasone equivalent doses ranged from $1320 \pm 465$ (Spain) to $570 \pm 497 \mu \mathrm{g} \cdot \mathrm{day}^{-1}$ (Italy).

Figures 3 and 4 provide an overview of maintenance therapy for severe asthma patients before starting with anti-IL-5 biologicals (mepolizumab, reslizumab or benralizumab; $\mathrm{n}=617$ ) and anti-IgE biologicals (omalizumab; $\mathrm{n}=514$ ) (see supplementary material for further details). In most of the registries, all patients 
TABLE 1 Baseline characteristics of patients included in different severe asthma registries that are part of SHARP

\begin{tabular}{|c|c|c|c|c|c|c|c|c|c|c|c|}
\hline & UK & Belgium & Italy & Spain & Netherlands & Germany & Poland & Slovenia & Hungary & Denmark & Sweden \\
\hline Patients & 765 & 629 & 437 & 410 & 237 & 209 & 193 & 140 & 130 & 59 & 27 \\
\hline Age years & $47.6 \pm 14.5$ & $56.9 \pm 14.7$ & $54.1 \pm 13.7$ & $56.4 \pm 14.8$ & $52.8 \pm 14.2$ & $44.4 \pm 20.4$ & $48.4 \pm 14.6$ & $53.5 \pm 12.8$ & $58.3 \pm 12.9$ & $51.9 \pm 16.1$ & $50.0 \pm 8.6$ \\
\hline Male & 285 (37.3) & $265(42.1)$ & $183(41.9)$ & $133(32.4)$ & $112(47.3)$ & $98(46.9)$ & $76(39.4)$ & $42(30.0)$ & 42 (32.3) & 28 (47.5) & 14 (51.9) \\
\hline \multicolumn{12}{|l|}{ Smoking status } \\
\hline Current smoker & $30(4.1)$ & $60(9.5)$ & $12(2.7)$ & $29(7.1)$ & $2(0.9)$ & $5(2.4)$ & $0(0)$ & $1(0.7)$ & $8(6.2)$ & $2(4.0)$ & $0(0)$ \\
\hline Never-smoker & $526(71.7)$ & $368(58.5)$ & 352 (80.5) & $281(68.5)$ & $136(57.9)$ & $125(59.8)$ & $171(88.6)$ & $92(65.7)$ & $108(83.1)$ & $24(48.0)$ & 16 (59.3) \\
\hline Ex-smoker & $178(24.3)$ & $201(32.0)$ & 73 (16.7) & $100(24.4)$ & $97(41.3)$ & 79 (37.8) & $22(11.4)$ & 47 (33.5) & $14(10.8)$ & $19(38.0)$ & $11(40.7)$ \\
\hline Pack-years & $15(5-20)$ & $15(6-27)$ & $9(4-15)$ & $19(10-23)$ & $10(4-19)$ & $8(2-15)$ & 12.5 (15) & $10(3-20)$ & $<5$ & $8.5(2.2-15)$ & $5(4-9)$ \\
\hline BMI $\mathbf{k g} \cdot \mathrm{m}^{-2}$ & $30.6 \pm 7.4$ & $27.7 \pm 12.6$ & $26.2 \pm 5.0$ & $28.2 \pm 6.0$ & $28.3 \pm 5.4$ & $27.4 \pm 10.8$ & 28.1 & $27.1 \pm 5.8$ & $26.9 \pm 5.4$ & $27.1 \pm 5.4$ & $27.7 \pm 5.3$ \\
\hline FEV $_{1} \%$ pred & $67.8 \pm 22.8$ & $67.9 \pm 21.6$ & $71.4 \pm 20.2$ & $68.1 \pm 36.1$ & $76.9 \pm 22.2$ & $70.3 \pm 23.0$ & $63.2 \pm 23.5$ & $69.6 \pm 19.8$ & $56.0 \pm 16.8$ & $72.0 \pm 19.1$ & $66.0 \pm 19.9$ \\
\hline FVC \% pred & $85.3 \pm 19.8$ & $88.2 \pm 20.2$ & $88.2 \pm 21.1$ & NA & $98.3 \pm 20.5$ & $84.9 \pm 19.7$ & NA & $95.0 \pm 15.6$ & $76.6 \pm 18.5$ & $78.2 \pm 18.3$ & $86.8 \pm 19.6$ \\
\hline $\begin{array}{l}\text { Eosinophils } \\
\times 10^{9} \mathrm{~L}^{-1}\end{array}$ & $\begin{array}{c}0.300 \\
(0.200-0.600)\end{array}$ & $\begin{array}{c}0.280 \\
(0.114-0.539)\end{array}$ & $\begin{array}{c}0.540 \\
(0.190-0.645)\end{array}$ & $\begin{array}{c}0.310 \\
(0.100-0.530)\end{array}$ & $\begin{array}{c}0.290 \\
(0.105-0.570)\end{array}$ & $\begin{array}{c}0.230 \\
(0.100-0.580)\end{array}$ & $\begin{array}{c}0.410 \\
(0.200-0.740)\end{array}$ & $\begin{array}{c}0.260 \\
(0.120-0.440)\end{array}$ & $\begin{array}{c}0.345 \\
(0.140-0.578)\end{array}$ & $\begin{array}{c}0.250 \\
(0.100-0.600)\end{array}$ & $\begin{array}{c}0.800 \\
(0.600-1.000)\end{array}$ \\
\hline $\begin{array}{l}\text { Neutrophils } \\
\times 10^{9} \mathrm{~L}^{-1}\end{array}$ & NA & NA & $\begin{array}{c}4.75 \\
(3.12-5.66)\end{array}$ & NA & $\begin{array}{c}5.48 \\
(4.09-7.25)\end{array}$ & $\begin{array}{c}5.02 \\
(3.71-7.15)\end{array}$ & NA & $\begin{array}{c}5.16 \\
(3.60-6.80)\end{array}$ & $\begin{array}{c}5.26 \\
(3.98-7.37)\end{array}$ & NA & $\begin{array}{c}3.80 \\
(2.90-5.10)\end{array}$ \\
\hline Total IgE IU.mL $\mathrm{mL}^{-1}$ & $\begin{array}{c}165 \\
(55.0-491)\end{array}$ & $\begin{array}{c}190 \\
(68.0-513)\end{array}$ & $\begin{array}{c}272 \\
(122-561)\end{array}$ & $\begin{array}{c}236 \\
(102-516)\end{array}$ & $\begin{array}{c}144 \\
(49-368)\end{array}$ & $\begin{array}{c}197 \\
(78.0-579)\end{array}$ & $\begin{array}{l}167.5 \\
(359)\end{array}$ & $\begin{array}{c}238 \\
(115-358)\end{array}$ & $\begin{array}{c}164 \\
(54.7-385)\end{array}$ & $\begin{array}{c}164 \\
(74.3-283)\end{array}$ & $\begin{array}{c}275 \\
(115-820)\end{array}$ \\
\hline$F_{\text {ENO }} \mathrm{ppb}$ & $41(23-77)$ & $25(14-42)$ & $32(17-64)$ & $33(19-52)$ & $33(20-60)$ & $33(19-79)$ & $27(27)$ & $66(27-101)$ & $32(18-56)$ & $26(13-49)$ & $57(29-80)$ \\
\hline Adult-onset asthma & $385(59.8)$ & $200(31.8)$ & $105(24.0)$ & NA & $129(63.2)$ & $61(39.4)$ & 68 (35.2) & $110(78.6)$ & $89(68.5)$ & 26 (78.8) & $24(88.9)$ \\
\hline$A C Q$ & $3.0 \pm 1.3$ & $2.5 \pm 1.3$ & $2.9 \pm 1.5$ & NA & $2.1 \pm 1.2$ & $2.6 \pm 1.5$ & $3.3 \pm 0.9$ & NA & NA & $2.4 \pm 1.4$ & $1.8 \pm 1$ \\
\hline АCT & NA & $13.2 \pm 5.4$ & $17.2 \pm 5.4$ & $15.9 \pm 5.8$ & NA & $15 \pm 6.0$ & 12.3 & $16.7 \pm 5.5$ & $16.6 \pm 1.2$ & NA & $12 \pm 3.8$ \\
\hline $\begin{array}{l}\text { Uncontrolled based } \\
\text { on ACQ/ACT }\end{array}$ & $581(84.6)$ & $331(76.1)$ & $250(54.6)$ & 221 (68.8) & 88 (61.5) & $135(71.1)$ & 191 (98.9) & $87(64.0)$ & $130(100)$ & $21(63.6)$ & $27(100)$ \\
\hline $\begin{array}{l}\text { Hospitalisation in } \\
\text { last year }\end{array}$ & 291 (39.5) & $229(36.4)$ & $53(17.6)$ & $52(12.7)$ & NA & $55(32.4)$ & $78(40.4)$ & $61(43.6)$ & $36(27.7)$ & $16(48.5)$ & $1(3.7)$ \\
\hline \multicolumn{12}{|l|}{ Included in: } \\
\hline Primary care & $0(0)$ & $0(0)$ & $0(0)$ & $16(3.9)$ & $0(0)$ & NA & $0(0)$ & $0(0)$ & $0(0)$ & $0(0)$ & $0(0)$ \\
\hline Secondary care & $0(0)$ & $52(8.3)$ & $0(0)$ & $125(30.5)$ & $76(32.1)$ & NA & $0(0)$ & $0(0)$ & $44(33.8)$ & $0(0)$ & $0(0)$ \\
\hline Tertiary care & $765(100)$ & 577 (91.7) & $437(100)$ & $269(65.6)$ & $161(67.9)$ & NA & $193(100)$ & $139(100)$ & 86 (66.2) & $59(100)$ & $27(100)$ \\
\hline $\begin{array}{l}\text { GINA step } 4 \\
\text { treatment }\end{array}$ & $162(21.2)$ & 309 (49.0) & $18(5.7)$ & $197(48.1)$ & $113(47.7)$ & $130(62.2)$ & 53 (27.5) & $87(62.6)$ & 93 (71.5) & $24(49.0)$ & $15(55.6)$ \\
\hline $\begin{array}{r}\text { GINA step } 5 \\
\text { treatment }\end{array}$ & $569(74.4)$ & $320(51.0)$ & 297 (94.3) & $210(51.2)$ & $118(49.8)$ & 79 (37.8) & $140(72.5)$ & $52(37.4)$ & 37 (28.5) & $14(28.6)$ & $12(44.4)$ \\
\hline \multicolumn{12}{|l|}{ Biological use } \\
\hline Total & $479(64.5)$ & $160(25.0)$ & 215 (49.2) & $210(51.2)$ & $82(34.6)$ & 80 (38.3) & $137(71.0)$ & 66 (47.5) & $30(23.1)$ & $59(100)$ & $0(0)$ \\
\hline Anti-lgE & $115(25.4)$ & $130(21.0)$ & $180(41.2)$ & $197(48.1)$ & 29 (12.2) & $41(19.6)$ & $129(66.8)$ & 59 (42.4) & 16 (12.3) & 18 (30.5) & $0(0)$ \\
\hline Anti-IL-5 & $337(74.4)$ & $30(5.0)$ & $35(8.0)$ & $13(3.2)$ & 53 (22.4) & 39 (18.7) & $8(4.1)$ & $7(5.0)$ & $14(10.8)$ & 41 (69.5) & $0(0)$ \\
\hline Anti-IL-4/IL-13 & $1(0.2)$ & $0(0)$ & NA & $0(0)$ & $0(0)$ & NA & $0(0)$ & $0(0)$ & $0(0)$ & $0(0)$ & $0(0)$ \\
\hline Thermoplasty & $0(0)$ & $9(1.4)$ & NA & $0(0)$ & $3(1.3)$ & NA & $0(0)$ & $2(1.4)$ & $0(0)$ & $0(0)$ & $0(0)$ \\
\hline $\begin{array}{l}\text { High-altitude } \\
\text { treatment }\end{array}$ & NA & $0(0)$ & NA & $0(0)$ & $33(14)$ & NA & $0(0)$ & $0(0)$ & $0(0)$ & NA & $0(0)$ \\
\hline $\mathrm{ICS}^{\#}$ dose $\mu \mathrm{g} \cdot \mathrm{day}^{-1}$ & $934 \pm 449$ & $954 \pm 501$ & $542 \pm 489$ & $1225 \pm 445$ & $1027 \pm 737$ & $676 \pm 398$ & $1220 \pm 668$ & $491 \pm 163$ & $920 \pm 370$ & $1073 \pm 372$ & $1196 \pm 641$ \\
\hline $\begin{array}{l}\text { OCS dose } \\
\mathrm{mg} \cdot \text { day }^{-1}\end{array}$ & $\begin{array}{c}10.0 \\
(10.0-20.0)\end{array}$ & $\begin{array}{c}9.0 \\
(5.0-10.0)\end{array}$ & $\begin{array}{c}10.7 \\
(5.0-20.0)\end{array}$ & $\begin{array}{c}10.0 \\
(5.0-10.0)\end{array}$ & $\begin{array}{c}10.0 \\
(5.0-17.5)\end{array}$ & $\begin{array}{c}10.0 \\
(5.0-15.0)\end{array}$ & $\begin{array}{c}7.0 \\
(7.0-15.0)\end{array}$ & $\begin{array}{c}10.0 \\
(5.0-10.0)\end{array}$ & $\begin{array}{c}7.5 \\
(5.0-10.0)\end{array}$ & NA & $\begin{array}{c}10.0 \\
(7.5-10.0)\end{array}$ \\
\hline
\end{tabular}

Data are presented as $n$, mean $\pm \mathrm{SD}, \mathrm{n}(\%)$ or median (interquartile range). Discrepancies between $\mathrm{n}(\%)$ and total number of patients are due to missing data. BMI: body mass index; FEV $\mathrm{V}_{1}$ forced expiratory volume in $1 \mathrm{~s}$; FVC: forced vital capacity; $F_{\text {ENO }}$ : exhaled nitric oxide fraction; ACQ: Asthma Control Questionnaire; ACT: Asthma Control Test; GINA: Global Initiative for Asthma; IL: interleukin; ICS: inhaled corticosteroid; OCS: oral corticosteroid; NA: not available. \#: dose expressed as fluticasone equivalent; ${ }^{\text {?: }}$ dose expressed as prednisone equivalent. 
TABLE 2 Medication prior to starting (or >6 months after stopping) high-cost therapy (biologicals, bronchial thermoplasty and high-altitude treatment)

\begin{tabular}{|c|c|c|c|c|c|c|c|c|c|c|c|}
\hline & UK & Belgium & Italy & Spain & Poland & Netherlands & Hungary & Slovenia & Denmark & Sweden & Germany \\
\hline Patients & 477 & 469 & 219 & 210 & 193 & 143 & 100 & 73 & 49 & 27 & NA \\
\hline GINA step 4 & $152(31.9)$ & $347(74.0)$ & $2(2.0)$ & $166(79.0)$ & 53 (27.5) & 54 (37.8) & 74 (74.0) & $42(57.5))$ & 24 (49.0) & $15(55.6)$ & NA \\
\hline GINA step 5 & $325(68.1)$ & 109 (23.2) & 98 (98.0) & $44(20.9)$ & $140(72.5)$ & 87 (60.8) & $26(26.0)$ & 31 (42.5) & $14(28.6)$ & $12(44.4)$ & NA \\
\hline SABA & 444 (93.3) & 377 (90.2) & $4(4.0)$ & 32 (15.2) & $193(100)$ & $107(74.8)$ & $100(100)$ & 43 (58.9) & 29 (59.2) & $27(100)$ & NA \\
\hline SAMA & NA & NA & NA & 175 (83.3) & 38 (19.7) & 25 (17.5) & $0(0)$ & 28 (38.3) & $1(2.0)$ & $8(29.6)$ & NA \\
\hline LABA & 436 (92.2) & $457(97.4)$ & $219(100)$ & 206 (98.1) & $155(80.3)$ & 135 (94.4) & $100(100)$ & $72(98.6)$ & 43 (87.8) & $27(100)$ & NA \\
\hline LAMA & $269(56.4)$ & $113(24.1)$ & $40(40.4)$ & 35 (28.9) & $37(19.2)$ & $42(29.4)$ & $14(14.0)$ & 54 (74.0) & $22(44.9)$ & $8(29.6)$ & NA \\
\hline ICS & 477 (100) & $457(97.4)$ & $219(100)$ & $212(100)$ & $193(100)$ & $142(99.3)$ & $100(100)$ & $73(100)$ & 46 (93.9) & $27(100)$ & NA \\
\hline ICS dose $^{\#} \mu \mathrm{g} \cdot$ day $^{-1}$ & $973 \pm 508$ & $986 \pm 479$ & $570 \pm 497$ & $1320 \pm 465$ & $1220 \pm 668$ & $1178 \pm 797$ & $909 \pm 386$ & $700.1 \pm 207.4$ & $1073 \pm 372$ & $1196 \pm 641$ & NA \\
\hline LTRA & $188(39.4)$ & 251 (53.5) & 37 (37.4) & $123(58.6)$ & $128(66.3)$ & 29 (20.3) & $45(45.0)$ & 30 (41.1) & 28 (57.1) & 21 (77.8) & NA \\
\hline Theophylline & $120(25.2)$ & $65(13.9)$ & $7(7.1)$ & $22(11.2)$ & $52(26.9)$ & $6(4.2)$ & 35 (35.0) & $1(1.4)$ & $5(10.2)$ & $2(7.4)$ & NA \\
\hline 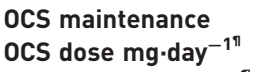 & $325(68.1)$ & $102(21.7)$ & 105 (71.9) & $45(21.4)$ & $87(45.1)$ & $88(60.8)$ & $26(26.0)$ & $31(42.5)$ & NA & $17(63.0)$ & NA \\
\hline Defined daily dose & NA & NA & NA & $1.81 \pm 0.89$ & NA & $1.4 \pm 1.1$ & $0.67 \pm 0.34$ & NA & NA & $0.94 \pm 0.29$ & NA \\
\hline Median (IQR) & $10.0(10.0-20.0)$ & $10.0(5.0-10.0)$ & $10.0(5.0-10.0)$ & $10.0(5.0-10.0)$ & $7.0(7.0-15.0)$ & $10.0(7.5-19.4)$ & $6.9(3.4-10.0)$ & $10.0(5.0-12.0)$ & NA & $10.0(7.5-10.0)$ & NA \\
\hline NSAID & NA & $25(5.0)$ & NA & NA & $44(22.8)$ & $3(2.2)$ & $0(0)$ & NA & NA & $20(74.1)$ & NA \\
\hline
\end{tabular}

Data are presented as $\mathrm{n}, \mathrm{n}(\%)$ or mean \pm SD, unless otherwise stated. Discrepancies between $\mathrm{n}$ (\%) and total number of patients are due to missing data. GINA: Global Initiative for asthma; SABA: short-acting $\beta$-agonist; SAMA: short-acting muscarinic antagonist; LABA: long-acting $\beta$-agonist; LAMA: long-acting muscarinic antagonist; ICS: inhaled corticosteroid;

LTRA: leukotriene receptor antagonist; OCS: oral corticosteroid; IQR: interquartile range; NSAID: nonsteroidal anti-inflammatory drug; NA: not available. \#: dose expressed as fluticasone equivalent; ๆ: dose expressed as prednisone equivalent.

\begin{tabular}{|c|c|c|c|c|c|c|c|c|c|c|c|}
\hline & UK & Belgium & Spain & Poland & Netherlands & Hungary & Slovenia & Denmark & Sweden & Italy & Germany \\
\hline \multicolumn{12}{|l|}{$\begin{array}{l}\text { Before starting } \\
\text { anti-IL-5 }\end{array}$} \\
\hline $\begin{array}{l}\text { Blood eosinophils } \\
\times 10^{9} \mathrm{~L}^{-1}\end{array}$ & $\begin{array}{c}0.400 \\
(0.300-0.700)\end{array}$ & $\begin{array}{c}0.490 \\
(0.320-0.760)\end{array}$ & $\begin{array}{c}0.530 \\
(0.330-0.830)\end{array}$ & $\begin{array}{c}0.450 \\
(0.405-0.855)\end{array}$ & $\begin{array}{c}0.360 \\
(0.165-0.610)\end{array}$ & $\begin{array}{c}0.685 \\
(0.233-1.010)\end{array}$ & $\begin{array}{c}0.440 \\
(0.280-0.670)\end{array}$ & $\begin{array}{c}0.27 \\
(0.100-0.600)\end{array}$ & $\begin{array}{c}0.800 \\
(0.600-1.000)\end{array}$ & NA & NA \\
\hline $\begin{array}{l}\text { Serum total } \\
\qquad \lg E \text { IU. } \mathrm{mL}^{-1}\end{array}$ & $129(44-404)$ & $270(90-376)$ & $431(168-594)$ & $405(360-791)$ & $140(48-366)$ & $56(29-200)$ & $149(53-256)$ & 164 (58-342) & $275(115-820)$ & NA & NA \\
\hline & $54(31-81)$ & 43 (32-48) & $48(40-70)$ & $27(20-47)$ & $36(24-60)$ & 52 (34-135) & $89.5(55-101)$ & $38(30-56)$ & $57(29-80)$ & NA & NA \\
\hline \multicolumn{12}{|l|}{$\begin{array}{l}\text { Before starting } \\
\text { anti-lgE }\end{array}$} \\
\hline $\begin{array}{l}\text { Blood eosinophils } \\
\times 10^{9} \mathrm{~L}^{-1}\end{array}$ & $\begin{array}{c}0.300 \\
(0.100-0.500)\end{array}$ & $\begin{array}{c}0.250 \\
(0.166-0.310)\end{array}$ & $\begin{array}{c}0.420 \\
(0.200-0.600)\end{array}$ & $\begin{array}{c}0.220 \\
(0.100-0.510)\end{array}$ & NA & $\begin{array}{c}0.210 \\
(0.150-0.438)\end{array}$ & $\begin{array}{c}0.235 \\
(0.150-0.370)\end{array}$ & $\begin{array}{c}0.130 \\
(0.100-0.300)\end{array}$ & NA & NA & NA \\
\hline $\begin{array}{l}\text { Serum total } \\
\qquad \lg E \text { IU. } \mathrm{mL}^{-1}\end{array}$ & 324 (139-567) & $238(107-626)$ & $243(114-515)$ & 154 (74-388) & NA & 172 (118-233) & 118 (32-795) & $148(92-228)$ & NA & NA & NA \\
\hline$F_{\text {ENO }} \mathrm{ppb}$ & 39 (24-82) & $29(16-41)$ & $36(20-57)$ & NA & NA & 34 (27-80) & 75 (41-92) & $10(12-30)$ & NA & NA & NA \\
\hline
\end{tabular}




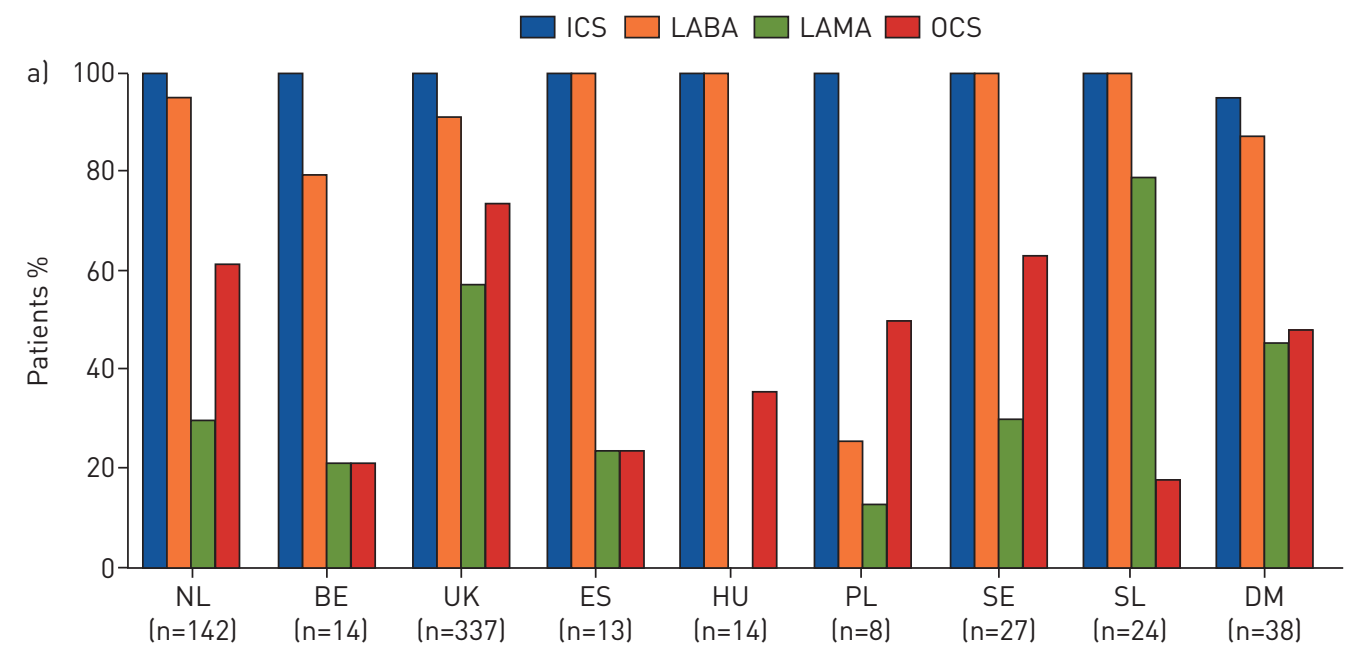

b)

c)
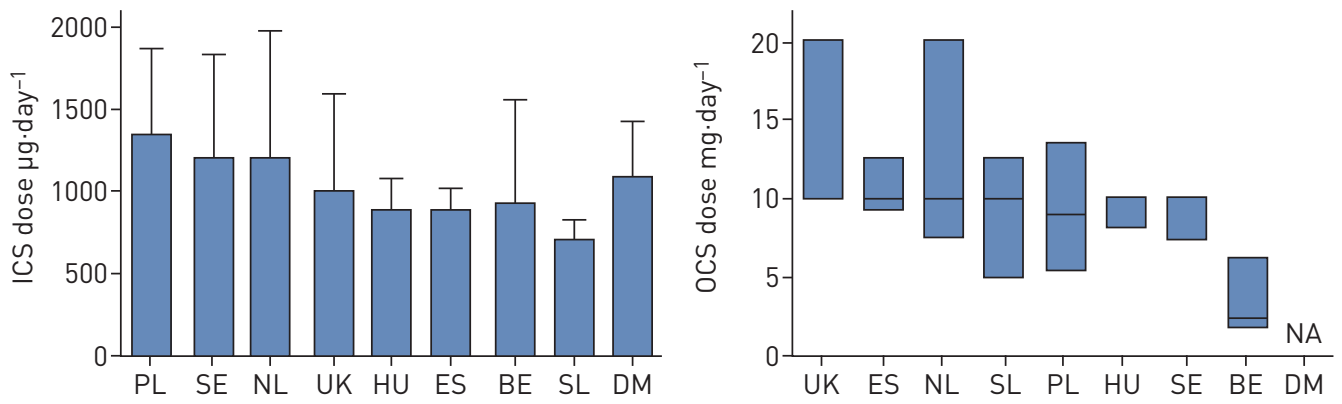

FIGURE 3 a) Overview of maintenance treatment of patients that start with anti-interleukin (IL)-5 biologicals. NL: Netherlands; BE: Belgium; ES: Spain; HU: Hungary; PL: Poland: SE: Sweden; SL: Slovenia; DM: Denmark; ICS: inhaled corticosteroid; LABA: long-acting $\beta$-agonist; LAMA: long-acting muscarinic antagonist; OCS: oral corticosteroid; NA: not available. b) Mean \pm SD ICS dose (fluticasone equivalent) in patients that start with anti-IL-5 biological therapies. c) Median (interquartile range) maintenance OCS dose (prednisone equivalent) in patients that start with anti-IL-5 biological therapies. Median values: UK $10 \mathrm{mg} \cdot \mathrm{day}^{-1}$, Spain $12.5 \mathrm{mg} \cdot$ day $^{-1}$, Netherlands $10 \mathrm{mg} \cdot \mathrm{day}^{-1}$, Slovenia $10 \mathrm{mg} \cdot \mathrm{day}^{-1}$, Poland $9 \mathrm{mg} \cdot \mathrm{day}^{-1}$, Hungary $10 \mathrm{mg} \cdot \mathrm{day}^{-1}$, Sweden $10 \mathrm{mg} \cdot \mathrm{day}^{-1}$ and Belgium $2.5 \mathrm{mg} \cdot \mathrm{day}^{-1}$.

were on ICS before starting anti-IL-5 (in Denmark this was only 94.7\%), although there were differences in ICS dose, ranging from $1335 \pm 529$ (Poland) to $700 \pm 118 \mu \mathrm{g} \cdot$ day $^{-1}$ (Slovenia). Information on treatment before starting omalizumab was available in six registries. ICS use was $<100 \%$ in Belgium (96.6\%) and Denmark (90.9\%), and the mean \pm SD dose ranged from $1344 \pm 540$ (Spain) to $772 \pm 191 \mu \mathrm{g} \cdot$ day $^{-1}$ (Slovenia). In four registries, all patients were using LABA before starting anti-IL-5 (Spain, Hungary, Sweden and Slovenia), while in other countries this ranged from $25.0 \%$ (Poland) to $94.4 \%$ (the Netherlands). LAMA use in patients starting anti-IL-5 ranged from 0\% (Hungary) to 79.1\% (Slovenia). LAMA use in patients starting anti-IgE starting ranged from $12.5 \%$ (Hungary) to $100 \%$ (Slovenia). OCS was used as a maintenance therapy before starting with anti-IL-5 in all registries and ranged from $21.0 \%$ (Belgium) to $63.0 \%$ (Sweden) of the population. Before starting anti-IgE, OCS was also used in all registries, ranging from $9.1 \%$ (Denmark) to $56.1 \%$ (the UK) of the population.

Table 3 shows differences between registries in biomarkers before starting treatment with biologicals. The median blood eosinophil levels before starting anti-IL-5 therapy were higher than levels seen before starting anti-IgE treatment in all registries. The median blood eosinophil levels before starting anti-IL-5 treatment ranged from $0.270 \times 10^{9}$ (Denmark) to $0.800 \times 10^{9}$ cells $\cdot \mathrm{L}^{-1}$ (Sweden). The median serum total $\operatorname{IgE}$ concentrations before starting with anti-IgE treatment ranged from 118 (Slovenia) to $324 \mathrm{IU} \cdot \mathrm{mL}^{-1}$ (the UK).

\section{Discussion}

This first collaborative study in the SHARP CRC has made several important observations. Across Europe there are large differences in characteristics and lifestyle factors of patients with severe asthma. Treatment regimens and biomarkers in patients starting biologicals and criteria for their prescription also seem to differ between countries. The patients included in the various countries of Europe for treatment with 
ICS $\square$ LABA $\square$ LAMA $\square$ OCS

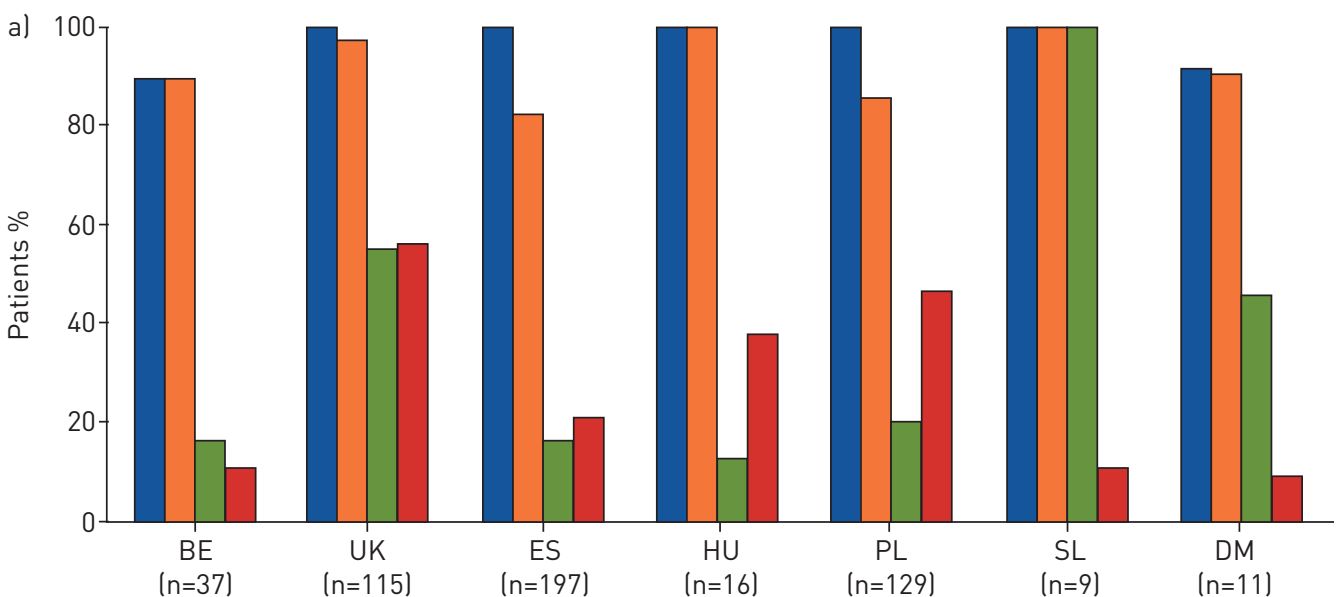

b)

c)
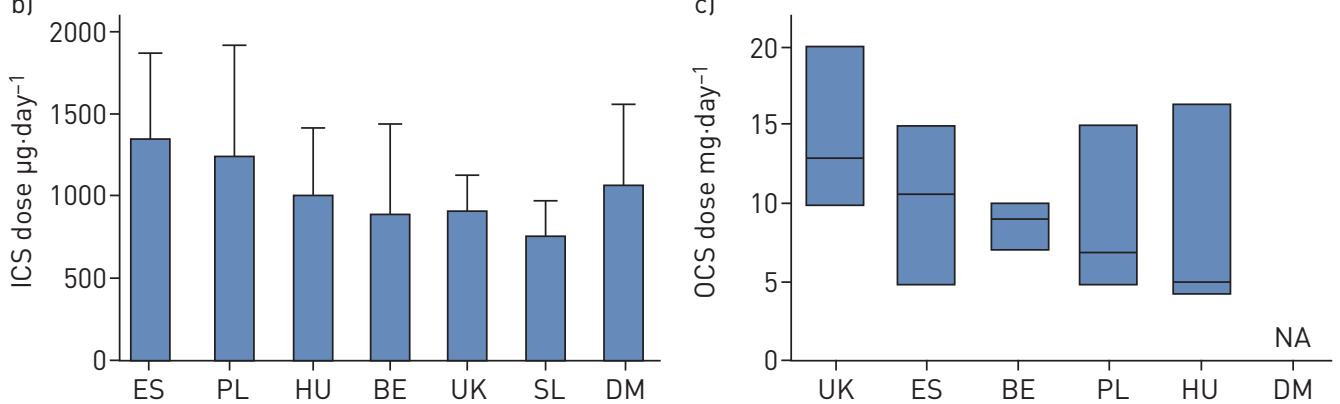

FIGURE 4 a) Overview of maintenance treatment of patients that start with anti-IgE biologicals. BE: Belgium; ES: Spain; HU: Hungary; PL: Poland: SL: Slovenia; DM: Denmark; ICS: inhaled corticosteroid; LABA: long-acting $\beta$-agonist; LAMA: long-acting muscarinic antagonist; OCS: oral corticosteroid; NA: not available. b) Mean \pm SD ICS dose (fluticasone equivalent) in patients that start with anti-IgE biological therapies. c) Median (interquartile range) maintenance OCS dose (prednisone equivalent) in patients that start with anti-lgE biological therapies. Median values: UK $13 \mathrm{mg} \cdot$ day $^{-1}$, Spain $10.7 \mathrm{mg} \cdot \mathrm{day}^{-1}$, Belgium $9 \mathrm{mg} \cdot \mathrm{day}^{-1}$, Poland $7 \mathrm{mg} \cdot$ day $^{-1}$ and Hungary $5 \mathrm{mg} \cdot \mathrm{day}^{-1}$.

biological therapies, who we would consider to suffer from severe asthma, did not fit the criteria of the definition of severe asthma as defined by ERS/ATS and GINA, and they also did not meet the criteria used to recruit patients in the phase 3 trials of these biological therapies. The reasons for these differences are as yet unclear and will need to be addressed as the SHARP CRC moves to harmonise the data that are collected in the different national registries.

\section{Differences between registries}

The data in this study clearly show large variation in the baseline characteristics of asthmatic subjects enrolled in the 11 European registries. This could be due to differences in the definition of severe asthma across the different registries. The disparities could, in principle, also reflect differences in overall severity of the broader asthma population in each country; however, our data do not allow us to explore to what extent the enrolled patients reflect the general asthma population. Lung function results, expressed as pre-bronchodilator $\mathrm{FEV}_{1}$ and FVC (\% pred), were in both cases highest in the Netherlands and lowest in Hungary, with differences as high as $20.9 \%$ and $21.7 \%$, respectively. We do not presently know what causes these differences; patients in the two registries were on similar treatment, and $F_{\text {ENO }}$ levels and blood eosinophil counts were not different. However, possible explanations may be in differences in lifetime dose or onset of therapy with ICS, which would result in progressive loss of lung function, or in the difference in OCS use between Hungary and the Netherlands (60\% versus $26 \%$ before high-cost therapies) and the resulting effects on blood eosinophils and $F_{\mathrm{ENO}}$. Important differences were also found in the percentage of adult-onset asthma patients (64.9\%), a clinical phenotype of asthma that is known to be more severe than early-onset asthma [8]. Furthermore, the percentages of patients with uncontrolled asthma based on questionnaire scores (45.4\% difference) and asthma-related hospitalisation during the past 12 months (43.5\% difference) point to possible differences in the quality of care (e.g. access to specialist care). Other potential explanations might be exposure to asthma triggers such as outdoor and/or indoor pollution 
(including cigarette smoke) that may have resulted in worsening lung function. While these factors could not be assessed in the current analysis because relevant data were not collected, they could be the subject of future studies by the SHARP CRC.

Smoking patients or smokers with a history $\geqslant 10$ pack-years are almost never included in asthma trials due to the risk of confounding effects of smoking and the undesired inclusion of chronic obstructive pulmonary disease patients. In real-life, however, significant proportions of severe asthma patients also smoke, with rates $>4 \%$ found in Belgium, the UK, Spain and Hungary. Again, differences between registries from different countries were large, up to 30.7 percentage points for the percentage of never-smokers, with differences in median pack-years $>14$ years. Interestingly, differences in smoking do not necessarily reflect the differences in lung function; the Netherlands included more ex-smokers and median pack-years is higher than in Hungary despite patients in Hungary having worse lung functions. In general, BMI appeared to be less variable. Nevertheless, the largest difference here was $4.4 \mathrm{~kg} \cdot \mathrm{m}^{-2}$, and the difference in mean BMI between the UK $\left(30.6 \mathrm{~kg} \cdot \mathrm{m}^{-2}\right)$ and Italy $\left(26.2 \mathrm{~kg} \cdot \mathrm{m}^{-2}\right)$ suggests that obesity in severe asthma patients may be a significant problem in the UK, but not in Italy.

\section{Treatment of patients starting on high-cost therapies}

The differences in OCS use before starting high-cost therapies between registries from different countries were striking. The percentage of patients on maintenance OCS varied greatly (largest difference 50.5 percentage points between Italy and Spain), suggesting very different prescribing regimens across Europe. When specifically studying patients starting anti-IL-5 and omalizumab, large differences in treatment regimens were also found. OCS use in those starting anti-IL-5 treatment was highest in the UK and differed most from clinical practice in Belgium (52.2\% difference). As expected, all patients starting anti-IL-5 biologicals were using regular ICS, although there was marked variation in the daily dose, with fluticasone equivalent differences of up to $635 \mu \mathrm{g} \cdot \mathrm{day}^{-1}$ (Poland versus Slovenia). Why this is the case is unclear. Potential explanations, which will require focused study by the SHARP CRC, include cost of treatment and fear of high-dose treatment-related side-effects.

Patients starting omalizumab showed similar differences between registries, with largest differences in OCS use and mean dose between the UK and Belgium (45.1 percentage points and $10.0 \mathrm{mg} \cdot \mathrm{day}^{-1}$, respectively). All patients starting omalizumab were on ICS except for in Belgium and the largest fluticasone equivalent difference was $571 \mu \mathrm{g} \cdot \mathrm{day}^{-1}$ (Spain versus Slovenia). LAMA can be used as step-up treatment after GINA step 4 [9], and the results show that the percentage of patients on LAMA in both anti-IL-5 and anti-IgE therapies varied significantly. LAMA use was common in Slovenia, although this observation was based on a small sample size (24 patients starting anti-IL-5 antibody and nine patients starting omalizumab). Of note, LAMAs were hardly used in Hungary, Poland and Spain. Taken together, these differences in treatment suggest a difference in criteria applied (not necessarily required) to prescribe anti-IL-5 and anti-IgE biologicals.

\section{Deviations from guidelines and trial criteria}

An important issue that this study highlights is that criteria on which severe asthma is defined currently by international guidelines and those used in clinical trials with biologicals do not match clinical reality. For example, not all patients enrolled in the registries are on GINA step 4/5 treatment and ICS doses in patients starting with biologicals do not always correspond to those applied as inclusion criteria in trials and in the joint ERS/ATS criteria. Fluticasone equivalence of $>1000 \mu \mathrm{g} \cdot \mathrm{day}^{-1}$ (ex-actuator: $880 \mu \mathrm{g} \cdot \mathrm{day}^{-1}$ ) was an inclusion criterion for mepolizumab trials $[10,11]$ and doses $>1000 \mu \mathrm{g} \cdot \mathrm{day}^{-1}$ are considered high dose according to the ERS/ATS guidelines [3]. In this study, patients in several registries (Belgium, the UK, Spain, Hungary and Slovenia) were on mean doses $<1000 \mu \mathrm{g} \cdot \mathrm{day}^{-1}$, suggesting that a significant proportion of patients in the registries would not meet the mepolizumab trial inclusion criteria or do not meet the international ERS/ATS criteria for severe asthma. This deviation in ICS dose can be potentially explained by different interpretations between what is considered high-dose ICS by the ERS/ATS and GINA ( $>500 \mu \mathrm{g} \cdot \mathrm{day}^{-1}$ fluticasone equivalent). Additionally, for the Belgian registry, these data can be partially explained by the inclusion of a large number ( 25-30\%) of non-type 2 asthma patients, who may be less responsive to ICS. A similar picture arises with anti-IgE treatment. Mean fluticasone equivalent ICS doses before starting omalizumab were $<1000 \mu \mathrm{g} \cdot \mathrm{day}^{-1}$ in Belgium, the UK and Slovenia; thus, at least part of the population does not have severe asthma according to international ERS/ATS guidelines.

The first clear message that arises is the need for agreement between ERS/ATS guidelines and GINA, as the current differences in definitions are a cause for confusion among pulmonary physicians. One of the possible explanations of the differences between the characteristics of patients included in the severe asthma registries and the characteristics that were expected according to the ERS/ATS guidelines definition of severe asthma might suggest that some patients do not fulfil guideline criteria but are being considered 
as having severe asthma by clinical severe asthma experts. These differences will require more analysis, including the processes whereby biologicals are offered to patients. In the UK, the main criteria required by the National Institute of Clinical Excellence for both omalizumab and anti-IL-5 biologicals are the frequency of exacerbations (three or four exacerbations) in the previous 12 months or maintenance OCS [12-14], and these are implemented rigorously by the commissioning groups that regulate the use of biologicals. One plausible explanation for the observation in the UK is that the frequency of exacerbations is not used to define asthma severity. Furthermore, patients treated with biologicals in clinical practice do not always fulfil the criteria that were used for inclusion in the biological trials. Although this study was not designed to evaluate the efficacy of biological therapies, this suggests a need for observational studies targeting the efficacy of biologicals in patients who were not enrolled in trials that resulted in their approval. Such observational studies would provide more insight into the efficacy of biologicals in daily practice; however, the differences in countries as described here should be considered.

\section{European harmonisation}

The aforementioned issues raise awareness that severe asthma research needs to consider more the complexity and heterogeneity between different populations of chronic respiratory diseases. New discoveries will need large amounts of data that can only be collected in international consortia and therefore there is an urgent need to harmonise datasets on severe asthma across Europe. An international consensus needs to be reached on a minimal set of variables that should be collected in the national registries that take part in SHARP. When studying lung function, for example, all registries currently include information on pre-bronchodilator $\mathrm{FEV}_{1}$, but only nine out of 11 registries also record pre-bronchodilator FVC. Information on lung function reversibility is even less common, with post-bronchodilator $\mathrm{FEV}_{1}$ currently recorded in only seven registries. The bigger challenge, however, lies in the harmonisation of definitions of variables. The definitions for $\mathrm{FEV}_{1}$ and $\mathrm{FVC}$ are rather simple, whereas the definition for adherence to therapy is not straightforward. Countries retrieved these data in different ways: by checking prescription records, check-up by a dedicated asthma nurse, doctor's assessment or checking a database whether a patient was registered as showing "good compliance". Accordance on a minimal set of well-defined key variables is needed to increase the usability of the SHARP platform and should be the subject of future studies. The data collected in the current study should be a stepping-stone to start the discussion about more standardised practice for severe asthma care in Europe.

\section{Earlier research}

Several of the participating registries have already published analyses of their data [15-17]. The heterogeneity found in the current study is in line with the analyses of registries in Belgium, Italy and the UK, where differences in inflammatory characteristics [15] among patient populations were found even between centres in the same country [16] and differences in phenotype were identified [17]. Analyses of other international cohorts also show a marked heterogeneity across severe asthma patients. The pan-European U-BIOPRED (Unbiased BIOmarkers for the Prediction of REspiratory Disease outcomes) cohort has provided evidence for the existence of different phenotypes and endotypes of severe asthma as well as evidence for "cluster-migrating" patients [18]. The US SARP (Severe Asthma Research Program) cohort has also shown that heterogeneity exists even within clinical clusters [19]. Our present study further confirms that the severe asthma phenotype may be an oversimplification of the clinical reality and that different phenotypes with different therapeutic needs exist within the population of severe asthma patients currently viewed as a single group. Large differences in prevalence of severe asthma that have been described [20] support the idea that current guidelines may be ambiguous.

\section{Strengths/limitations}

This first ever attempt to integrate registry data across Europe has limitations. With over 3000 patients included in the analysis, it is one of the largest comparisons of this population to date, providing insight into the characteristics and treatments of this heterogeneous group across Europe. With representation from Southern, Western, Eastern and Northern Europe there is a good geographical distribution; thus, the influence of differences in environmental and genetic factors and in healthcare systems have been incorporated, but to what extent these influence the observed heterogeneity is unclear. Perhaps the biggest, but inevitable, weakness is the retrospective nature of the study. Indeed, there was significant variation in inclusion criteria and only half the registries used the joint ERS/ATS definition of severe asthma. Furthermore, not all patients were treated in a specialised asthma centre, while half the registries solely included patients in tertiary care, reflecting diversity in what clinicians in different European countries consider to be severe asthma. A further important limitation of the current data is pre-selection of specific patient subgroups; in particular, the registries in the Netherlands, Sweden and Slovenia focused on including patients that were starting biological therapies, which is expected to result in cohorts composed of the most severe patients. However, we expected this pre-selection of more severe 
patients to be reflected in a selection of patients who met the current international guidelines, but that was not the case. Some registries, i.e. Sweden, are currently run in only one city or even one hospital, which implies that data do not necessarily reflect a country, but sometimes a specific situation in a country.

\section{Conclusions}

In summary, this study shows that the population of severe asthma patients in Europe is heterogeneous and differs in both clinical characteristics and treatment. These results have several key implications. First, severe asthma populations and treatment, even when users of biologicals are excluded, greatly differ between countries. Thus, results from single-centre trials, or even multicentre trials in the same country, cannot necessarily be extrapolated to other countries. Second, the definition of severe asthma in current guidelines does not comply with the characteristics of real-world severe asthma patients and therefore there might be differences in the application of these guidelines in the different countries. Third, the first key messages underline the importance of harmonisation of severe asthma databases across Europe and the need for long-term follow-up of the patient. A consensus on the data that must be collected to provide solutions to these challenges should be agreed and this will provide a logical next step for the SHARP CRC. Of importance to future research in the SHARP CRC, the use of aggregated data proved to be a relatively easy way to obtain data that can be used for international collaboration.

Acknowledgements: The authors would like to thank Elise Heuvelin (Clinical Project Manager, ERS, Lausanne, Switzerland) for her much appreciated support in collecting the data.

Members of the SHARP Clinical Research Collaboration are: B. Abenhardt, Praxis Dr Abenhardt und Jochen Hinrichs-Pavlik, Heidelberg, Germany; I.M. Adcock, National Heart and Lung Institute, Imperial College London, London, UK; J. Adler, European Lung Foundation, Sheffield, UK; R. Alfonso, GSK, Durham, NC, USA; R. Ali, Barts Health NHS Trust, London UK; S. Alkameh, Lungenfachpraxis Backnang, Backnang, Germany; C. Almonacid Sánchez, Hospital Ramón y Cajal, Madrid, Spain; L. Alvares, Novartis Pharma AG, Basel, Switzerland; G. Anderson, University of Melbourne, Melbourne, Australia; K. Assing, Aalborg University Hospital, Aalborg, Denmark; S. Ayre, European Lung Foundation, Sheffield, UK; J. Becker, Facharztpraxis für Pneumologie, Lübeck, Germany; E.H.D. Bel, Amsterdam UMC, University of Amsterdam, Amsterdam, The Netherlands; K. Bergmann, Charité Berlin Allergie-Centrum, Berlin, Germany; K. Bieksiene, Lithuanian University of Health Science, Kaunas, Lithuania; N. Bjerring, Odense University Hospital, Odense, Denmark; F. Blasi, Milano Respiratory Unit and Adult Cystic Fibrosis Centre and University of Milan, Milan, Italy; P. Bloemen, GSK, Zeist, The Netherlands; H. Blum, MECS Dortmund GmbH, Dortmund, Germany; S. Böing, Pneumoplus, Lungen- und Allergiezentrum, Neuss, Germany; M. Bonavia, Respiratory Rehabilitation, ASL3, Genoa, Italy; A. Bossios, Karolinska University Hospital, Huddinge, Sweden; A. Bourdin, PhyMedExp, INSERM, EFS, Université de Montpellier, CHU Montpellier, Montpellier, France; G-J. Braunstahl, Sint Franciscus Gasthuis and Vlietland, Rotterdam, The Netherlands; A. Brons, European Lung Foundation, Sheffield, UK; G. Brusselle, University of Ghent, UZ Ghent, Ghent; J. Buis, Teva Pharmaceuticals, Amsterdam, The Netherlands; J. Busby, Queen's University Belfast, Belfast, UK; M. Caiaffa, University of Foggia, Foggia, Italy; C. Calabrese, University of Campania "L. Vanvitelli", Caserta, Italy; G. Camiciottoli, Careggi University Hospital, Florence, Italy; G.W. Canonica, Humanitas University, Rozzano and SANI-Severe Asthma Network Italy, Milan, Italy; H. Cao, Novartis Pharmaceuticals Corp., East Hanover, NJ, USA; C. Caruso, Fondazione Policlinico Universitario A. Gemelli, IRCCS, Rome, Italy; M. Castilla Martínez, Hospital Los Arcos del Mar Menor, Murcia, Spain; S. Centanni, Università degli Studi Milano, Respiratory Unit, ASST Santi Paolo e Carlo, Milan, Italy; K.F. Chung, National Heart and Lung Institute, Imperial College London, London, UK; C. Cisneros Serrano, Hospital de La Princesa, Madrid, Spain; A. Corsico, IRCCS Policlinico San Matteo Foundation and University of Pavia, Pavia, Italy; L. Cosmi, University of Florence, Florence, Italy; M. Costantino, "Carlo Poma" Hospital Mantova, Mantova, Italy; R. Costello, Royal College of Surgeons in Ireland, Beaumont Hospital, Dublin, Ireland; N. Crimi, University of Catania, Catania, Italy; Z. Csoma, National Koranyi Institute of Pulmonology, Budapest, Hungary; S. Dahlen, Karolinska Institutet, Stockholm, Sweden; B. Dahlén, Karolinska University Hospital, Huddinge, Sweden; M. D'Amato, "Federico II" University, AO Dei Colli, Naples, Italy; D. Davies, Southampton University Hospital, Southampton, UK; E. Davin, European Lung Foundation, Sheffield, UK; F. de Borja García-Cosío Piqueras, Hospital Son Espases Palma Mallorca, Islas Baleares, Spain; G. Decarlo, European Federation of Allergy and Airways Diseases (EFA), Brussels, Belgium; A. Deimling, Lungenpraxis Schleswig, Schleswig, Germany; S. Del Giacco, University of Cagliari, Cagliari, Italy; R. Diaz Campos, Hospital 12 Octubre, Madrid, Spain; M. Djandji, Medical Affairs, Sanofi Genzyme, Cambridge, MA, USA; R. Djukanovic, University of Southampton, Southampton, UK; D. Doberer, Vienna General Hospital, Vienna, Austria; L. Dupont, University of Leuven, UZ Gasthuisberg Leuven, Leuven, Belgium; K. Dyett, European Lung Foundation, Sheffield, UK; N. Edelbaher, University Clinical Centre Maribor, Maribor, Slovenia; M. Edelmann, Lungenpraxis Aalen, Aalen, Germany; R. Ehmann, Gemeinschaftspraxis für Ambulante Pneumologie mit Allergiezentrum, Stuttgart, Germany; A. Ekberg-Jansson, Dept of Research and Development, Region Halland and University of Gothenburg, Gothenburg, Sweden; A. Farsi, SOS of Allergology and Clinical Immunology, Prato, Azienda USL Toscana Centro, Italy; E. Favero, Vittorio Veneto Hospital, Treviso, Italy; J. Feimer, Pneumologie Odeonsplatz, München, Germany; M. Fletcher, GSK, Brentford, UK; B. Foschino, University of Foggia, Foggia, Italy; B. Frankemölle, European Lung Foundation, Sheffield, UK; M. Gaga, Athens Chest Hospital Sotiria, Athens, Greece; M. Gappa, Marien-Hospital, Klinik für Kinder- und Jugendmedizin, Wesel, Germany; J. García de Pedro, Hospital Gregorio, Marañón, Madrid, Spain; J. García Rivero, Hospital Laredo, Laredo, Spain; M. Gasplmayr, Kardiologische und Fachinternistische ÜBAG Dr Sandrock und Partner, Altdorf bei Nürnberg, Germany; R. Gebhardt, Facharzt für Lungenund Brochialheilkunde, Allergologie und Umweltmedizin, Berlin, Germany; H. Geldmacher, Pneumologicum, Hannover, Germany; C. Geltner, Kreisklinik Bad Reichenhall, Bad Reichenhall, Germany; M. Gerstlauer, Klinikum 
Augsburg, II Kinderklinik, Augsburg, Germany; T. Gibson, European Lung Foundation, Sheffield, UK; G. Giuseppe, AO S. Croce e Carle, Cuneo, Italy; C. Gogoll, Evangelische Elisabeth Klinik, Berlin, Germany; V. Grimm-Sachs, Praxis Dr Grimm-Sachs, Bruchsal, Germany; I. Grisle, Riga Eastern Clinical University Hospital, Riga, Latvia; B. Grün, Praxis Dr Grün, Bad Windsheim, Germany; A. Grünewaldt, Universitätsklinikum Frankfurt, Frankfurt, Germany; G. Guarnieri, University of Padua, Padova, Italy; J. Gullón Blanco, Hospital San Agustín, Avilés, Asturias, Spain; E. Hamelmann, Klinik für Kinder- und Jugendmedizin Kinderzentrum Bethel, Bielefeld, Germany; D. Hamerlijnck, European Lung Foundation, Sheffield, UK; A. Hammers-Reinhard, Praxis Hammers-Reinhard, Homburg-Saar, Germany; S. Hanon, Free University Brussel, Academic Ziekenhuis, Jette, Belgium; S. Hansen, Bispebjerg University Hospital, Copenhagen, Denmark; D. Harzheim, Waldburg-Zeil Kliniken - Fachkliniken Wangen, Wangen im Allgäu, Germany; L. Heaney, Queens University Belfast and Belfast Health and Social Care Trust, Belfast, UK; E. Heffler, Humanitas University, Rozzano and SANI-Severe Asthma Network Italy, Milan, Italy; S. Hellmich, Pneumologie am Schelztor Esslingen, Esslingen, Germany; M. Herden, Lungenfachärztlich-Internistische Schwerpunktpraxis, Freising, Germany; T. Hering, Arzt für Pneumologie, Allergologie, Schlafmedizin, Berlin, Germany; F. Herth, Thoraxklinik Heidelberg gGmbH, Heidelberg, Germany; O. Hilberg, Vejle Hospital, Vejle, Denmark; I. Horvath, National Koranyi Institute of Pulmonology, Budapest, Hungary; P. Howarth, GSK, Brentford, UK; M. Hubatsch, Lungenarztpraxis Dr Hubatsch, Heilbronn, Germany; M. Humbert, Université Paris-Sud, Le Kremlin-Bicêtre, France; K. Husemann, MVZ Klinikum Kempten, Praxis für Pneumologie und Allergologie, Kempten, Germany; M. Idzko, Klinik für Pneumologie, Universitätsklinikum Freiburg, Freiburg, Germany; D. Jackson, Guy's and St Thomas' NHS Trust and King's College London, London, UK; M. Jandl, Hamburger Institut für Therapieforschung GmbH, Hamburg, Germany; X. Jaumont, Novartis Pharma AG, Basel, Switzerland; G. Joos, UZ Gent, University of Gent, Belgium; M. Jöst, Malteser Lungen- und Allergiezentrum Bonn, Bonn, Germany; M. Jüch, Pneumologische Praxis am Ulrichplatz, Magdeburg, Germany; M. Kabesch, Krankenhaus Barmherzige Brüder Regensburg, Regensburg, Germany; P. Kaiser-Labusch, Klinikum Bremen Mitte, Bremen, Germany; P. Kardos, Studienzentrum Maingau, Frankfurt, Germany; F. Käßner, MECS Cottbus, Cottbus, Germany; T. Keeley, GSK, Brentford, UK; W. Kerr, GSK, Brentford, UK; J. Kirschner, CIMS Studienzentrum Bamberg, GmbH, Bamberg, Germany; L. Klimek, Zentrum für Rhinologie und Allergologie, Wiesbaden, Germany; M. Koca, Lungenpraxis Offenbach, Offenbach, Germany; R. Koczulla, Schönklinik Berchtesgadener Land, Schönau am Königsee, Germany; C. Koerner-Rettberg, Klinik für Kinderund Jugendmedizin der RUB im St Josef-Hospital, Bochum, Germany; P. Kopac, University Clinic of Respiratory and Allergic Diseases, Golnik, Slovenia; S. Korn, Universitätsmedizin Mainz, Mainz, Germany; S. Korn, Schwerpunkt Pneumologie, Universitätsmedizin Mainz, Mainz, Germany; M. Kots, Chiesi Farmaceutici, Parma, Italy; J. Kronsbein, Berufsgenossenschaftliches Universitätsklinikum Bergmannsheil, Bochum, Germany; P. Kuna, Medical University of Lodz, Lodz, Poland; I. Kupryś Lipinska, Medical University of Lodz, Poland; N. Kwon, GSK, Brentford, UK; M. Langer, Lungenpraxis Dr Langer Tübingen, Tübingen, Germany; B. Langeveld, Deventer Hospital, Deventer, The Netherlands; A. Lantz, Karolinska University Hospital, Huddinge, Sweden; N. Lazarinis, Karolinska University Hospital, Huddinge, Sweden; Z. Lazic, University Clinical Centre Kragujevac, Kragujevac, Serbia; L. Lehtimäki, University of Tampere, Tampere, Finland; J. Leuppi, University Clinic of Internal Medicine, Basel, Switzerland; C. Lombardi, Hospital Institute Fondazione Poliambulanza, Brescia, Italy; M. Lommatzsch, Universität Rostock, Abteilung Pneumologie, Rostock, Germany; A. López-Viña, Hospital Puerta Hierro, Majadahonda, Madrid, Spain; R. Louis, CHU Liege, GIGA-I3 Research Group, University of Liege, Liege, Belgium; R. Luca, Catholic University of the Sacred Heart, Fondazione Policlinico Universitario A. Gemelli, IRCCS, Rome, Italy; D. Lúðvíksdóttir, Landspitali University Hospital, Reykjavik, Iceland; C. Lüttecke-Hecht, Lungenfacharztpraxis Dr C. Lüttecke-Hecht, Mainz, Germany; L. Macchia, University of Bari - Aldo Moro, Bari, Italy; T. Magni, Chiesi Farmaceutici, Parma, Italy; A.H. Maitland-van der Zee, Amsterdam UMC, University of Amsterdam, Amsterdam, The Netherlands; C. Martínez Rivera, Hospital Germans Trias i Pujol, Badalona, Barcelona; P. Mastoridis, Novartis Pharmaceuticals Corp., East Hanover, NJ, USA; F. Mazza, Presidio Ospedaliero of Pordenone, Pordenone, Italy; F. Menzella, Santa Maria Nuova Hospital, Azienda USL di Reggio Emilia IRCCS, Reggio Emilia, Italy; A. Menzies-Gow, Royal Brompton Hospital and Imperial College London, London, UK; A. Michils, Hôpital Erasme, Bruxelles, Belgium; F. Mihălțan, University of Medicine and Pharmacy "Carol Davila", Bucharest, Romania; M. Milanese, Pulmonology Unit, ASL2 Savonese, Pietra Ligure, Savona, Italy; K. Milger-Kneidinger, Klinikum der Universität München, München, Germany; J. Molinska, Medical University of Lodz, Lodz, Poland; I. Montagna, Chiesi Farmaceutici, Parma, Italy; P. Montuschi, Catholic University of the Sacred Heart, Fondazione Policlinico Universitario A. Gemelli, IRCCS, Rome, Italy; N. Mülleneisen, Asthma und Allergiezentrum, Leverskusen, Germany; M. Muñoz Esquerre, Hospital Bellvitge, Barcelona, Spain; A. Nanzer-Kelly, Guy's and St Thomas' NHS Trust and King's College London, London, UK; N. Nenasheva, Russian Medical Academy for Postgraduate Education, Moscow, Russia; C. Neurohr, Klinik Schillerhöhe, Abteilung für Pneumologie und Beatmungsmedizin, Gerlingen, Germany; E. Nucera, Catholic University of the Sacred Heart, Fondazione Policlinico Universitario A. Gemelli, IRCCS, Rome, Italy; J. Otker, European Lung Foundation, Sheffield, UK; K. Oud, Hospital Gelderse Vallei, Ede, The Netherlands; P. Paggiaro, University of Pisa, Pisa, Italy; R. Parente, University of Salerno, Salerno, Italy; J. Parkinson, Asthma UK, London, UK; G. Passalacqua, IRCCS Policlinico San Martino, University of Genoa, Genoa, Italy; N. Patberg, Isala Hospital, Zwolle, The Netherlands; V. Patella, "Santa Maria della Speranza" Hospital, Battipaglia, Salerno, Italy; O. Patino, Teva Pharmaceuticals, Amsterdam, The Netherlands; T. Paulsson, GSK, Brentford, UK; R. Peche, Hôpital Vésale, Charleroi, France; G. Pelaia, University Magna Graecia of Catanzaro, Catanzaro, Italy; E. Peress, Novartis Pharma AG, Basel, Switzerland; L. Pérez de Llano, Hospital Lucus Augusti, Lugo, Spain; P. Pfeffer, Barts Health NHS Trust and Barts and The London School of Medicine and Dentistry, Queen Mary University of London, London, UK; P. Pfister, Novartis Pharma AG, Basel, Switzerland; C. Pilette, CHU Saint Luc, Université Catholique de Louvain, Belgium; C. Pinedo Sierra, Hospital San Carlos, Madrid, Spain; L. Pini, University of Brescia, Spedali Civili di Brescia, Brescia, Italy; V. Plaza, Hospital de la Santa Creu i Sant Pau, Universitat Autònoma de Barcelona, Barcelona, Spain; C. Porsbjerg, Bispebjerg University Hospital, Copenhagen, Denmark; F. Powitz, Pneumologie Elisenhof München, München, Germany; D. Ramos-Barbon, Hospital de la Santa Creu i Sant Pau, Universitat Autònoma de Barcelona, Barcelona, Spain; T. Ranger, European Lung Foundation, Sheffield, UK; L. Rasmussen, Copenhagen University Hospital Gentofte, Gentofte, Denmark; K. Rasmussen, Zealand University Hospital, Roskilde, Denmark M. Rezelj, University Clinic of Respiratory and Allergic Diseases, Golnik, Slovenia; L. Ricciardi, University Hospital "G. Martino", University of Messina, Italy; F. Ricciardolo, University of Torino, San Luigi Hospital, Orbassano, Torino, Italy; L. B. Richards, Amsterdam UMC, University of Amsterdam, Amsterdam, The Netherlands; E. Ridolo, University of Parma, Parma, Italy; L. Rijssenbeek-Nouwens, Dutch Asthma Centre Davos, Davos, Switzerland; G. Rolla, AO Mauriziano Hospital, University of Torino, Turin, Italy; D. Romero Ribate, Hospital La Paz, Madrid, Spain; S. Rüdiger, Universitätsklinikum Ulm, Ulm, Germany; G. Safioti, Teva Pharmaceuticals, Amsterdam, The Netherlands; T. Sandström, 
Umeå University, Umeå, Sweden; P. Santus, Università degli Studi di Milano, Sacco University Hospital, ASST Fatebenefratelli-Sacco, Milano, Italy; R. Sauer, Lungenzentrum Ulm, Ulm, Germany; G. Schauerte, CJD Berchtesgaden, Asthmazentrum und Diabeteszentrum, Berchtesgaden, Germany; R. Schipmann, Klinik Martinusquelle, Bad Lippspringe, Germany; F. Schleich, University of Liege, CHU Liege, Liege, Belgium; J. Schmid, Aarhus University Hospital, Aarhus, Denmark; F. Schmidt, Pneumologische Gemeinschaftspraxis Dr Schmidt und Weeg, München, Germany; O. Schmidt, Lungenfachärzte KSS, Koblenz, Germany; M. Schmitz, Pneumo Westpfalz, Kaiserslautern, Germany; T. Schrag, Praxis Dr med. Till Schrag, Bad Reichenhall, Germany; S. Schröer, Internistische Schwerpunktpraxis, Villingen-Schwenningen, Germany; K. Schultz, Klinik Bad Reichenhall, Bad Reichenhall, Germany; C. Schulz, Universitätsklinikum Regensburg, Regensburg, Germany; N. Scichilone, University of Palermo, Palermo, Italy; V. Sedlak, Czech Pneumology and Phthiseology Society, Prague, Czech Republic; J. Selb, University Clinic of Respiratory and Allergic Diseases, Golnik, Slovenia; G. Senna, University Hospital of Verona, Verona, Italy; S. Sergejeva, University of Tartu, Tartu, Estonia; J. Serrano Pariente, Hospital Inca, Islas Baleares, Spain; M. Sichau, MVZ für Diagnostik und Therapie, Herne, Germany; D. Simona, AV3 ASUR Marche, Hospital Civitanova Marche, Macerata, Italy; A. Singer, Barts Health NHS Trust, London UK; D. Skowasch, Universitätsklinikum Bonn, Bonn, Germany; S. Škrgat, University Clinic of Respiratory and Allergic Diseases, Golnik, Slovenia; F. Smeenk, Catharina Hospital, Eindhoven, The Netherlands; S. Smith, GSK, Durham, NC, USA; P. Solidoro, University of Turin, Turin, Italy; J.K. Sont, Leiden University Medical Centre, Leiden, The Netherlands; G. Spadaro, AO Universitaria Federico II, Naples, Italy; A. Spanevello, Istituti Clinici Scientifici Maugeri IRCCS, Tradate, Italy and University of Insubria, Varese, Italy; M. Stefansdottir, European Lung Foundation, Sheffield, UK; K. Steinmetz, Gemeinschaftspraxis, Darmstadt, Germany; J. Steiß, Universitätsklinikum Giessen, Giessen, Germany; M. Stephan, Klinik Löwenstein, Löwenstein, Germany; S. Stieglitz, Wuppertaler Lungenzentrum, Wuppertal, Germany; H. Suhling, MH Hannover, Hannover, Germany; C. Taube, Universitätsmedizin Essen, Westdeutsches Lungenzentrum am Universitätsklinikum Essen gGmbH, Essen, Germany; A. ten Brinke, Medical Centre Leeuwarden, Leeuwarden, The Netherlands; S. Tolga Yavuz, Universitätsklinikum Bonn, Bonn, Germany; N. Tudoric, Dubrava University Hospital, Zagreb, Croatia; C. Ulrik, Hvidovre University Hospital, Copenhagen, Denmark; J.J.M.H. van Bragt, Amsterdam UMC, University of Amsterdam, Amsterdam, The Netherlands; M. van de Ven, Rijnstate Hospital, Arnhem, The Netherlands; F. van den Elshout, Rijnstate Hospital, Arnhem, The Netherlands; M. Van Dyke, GSK,Collegeville, PA, USA; S. van Nederveen-Bendien, Haga Hospital, The Hague, The Netherlands; I. van Veen, Medisch Spectrum Twente, Enschede, The Netherlands; O. Vandenplas, CHU Godine Namur, Belgium; K. Velthove, GSK, Zeist, The Netherlands; A. Vianello, University of Padova, Padova, Italy; S.J.H. Vijverberg, Amsterdam UMC, University of Amsterdam, Amsterdam, The Netherlands; C. Vogelberg, Universitätsklinikum Carl Gustav Carus, Klinik und Poliklinik für Kinder- und Jugendmedizin, Dresden, Germany; S.S. Wagers, BioSciConsulting, Maasmechelen, Belgium; E. Wallén-Nielsen, Karolinska University Hospital, Huddinge, Sweden; E.J. Weersink, Amsterdam UMC, University of Amsterdam, Amsterdam, The Netherlands; T. Wisskirchen, Aeroprax, Wuppertal, Germany; M. Yacoub, San Raffaele Hospital of Milano, Milan, Italy; S. Yancey, GSK, Durham, NC, USA; V. Yasinska, Karolinska University Hospital, Huddinge, Sweden; M. Zappa, Sandro Pertini Hospital, Rome, Italy; S. Zielen, Universitätsklinikum Frankfurt, Frankfurt, Germany; C. Zimmermann, Pneumologische Praxis Reutlingen, Reutlingen, Germany; R. Zimmermann, Klinikum Landshut, Medizinische Klinik 2, Landshut, Germany.

Conflict of interest: J.J.M.H. van Bragt has nothing to disclose. I.M. Adcock has nothing to disclose. E.H.D. Bel reports grants and personal fees from AstraZeneca, GSK and Novartis, grants from Teva, and personal fees from Boehringer Ingelheim, Sanofi/Regeneron, Vectura and Sterna, outside the submitted work. G-J. Braunstahl reports grants from GSK, Novartis, AstraZeneca and Chiesi, outside the submitted work. A. Ten Brinke reports institutional fees for research advisory boards and lectures from GSK, Teva and AstraZeneca, institutional fees for research advisory boards from Sanofi, Novartis and Boehringer Ingelheim, outside the submitted work. J. Busby has nothing to disclose. G.W. Canonica reports personal fees from A. Menarini, AstraZeneca, Boehringer Ingelheim, Chiesi Farmaceutici, Circassia, GSK, MSD, Novartis, Roche, Sanofi-Aventis and Teva, during the conduct of the study. H. Cao is an employee of Novartis, which is one of the funding pharmaceutical companies of SHARP. K.F. Chung has received honoraria for participating in advisory board meetings from GSK, AstraZeneca, Novartis, Merck, Boehringer Ingelheim and Teva regarding treatments for asthma and chronic obstructive pulmonary disease and has also been remunerated for speaking engagements. Z. Csoma has nothing to disclose. B. Dahlén reports advisory board membership for Teva, GSK and Sanofi, personal fees for lectures from AstraZeneca, outside the submitted work. E. Davin has nothing to disclose. S. Hansen has nothing to disclose. E. Heffler reports personal fees from AstraZeneca, Sanofi Genzyme, Teva, Novartis, GSK, Circassia and Nestle Purina, outside the submitted work. I. Horvath reports personal fees from AstraZeneca, Boehringer Ingelheim, GSK, Chiesi, Berlin-Chemie, Roche, MSD, CSL and Sager Pharma, outside the submitted work. S. Korn reports personal fees from Almirall, AstraZeneca, Boehringer Ingelheim, Chiesi, Teva and Roche, grants and personal fees from GSK and Novartis, during the conduct of the study. M. Kots is a full time Chiesi Famaceutici SpA employee, Global clinical development department. P. Kuna reports personal fees for lectures and advisory board work from AstraZeneca and Novartis, personal fees for lectures and nonfinancial support for meeting attendance from Berlin-Chemie, Menarini and Boehringer Ingelheim, outside the submitted work. N. Kwon is an employee of GSK. R. Louis reports grants and personal fees for advisory board work from GSK, AstraZeneca and Novartis, grants from Chiesi, outside the submitted work. V. Plaza reports grants, personal fees and nonfinancial support from Chiesi, grants and personal fees from AstraZeneca, personal fees from ALK, Mundipharma and Sanofi, grants from Menarini, outside the submitted work. C. Porsbjerg has nothing to disclose. D. Ramos-Barbon has nothing to disclose. L.B. Richards has nothing to disclose. S. Škrgat reports personal fees for lectures and scientific discussion at symposia from Teva and Boehringer, personal fees for lectures and advisory boards from AstraZeneca, Glaxo, Berlin-Chemie and Chiesi, outside the submitted work. J.K. Sont has nothing to disclose. S.J.H. Vijverberg has nothing to disclose. E.J. Weersink has nothing to disclose. V. Yasinska has nothing to disclose. S.S. Wagers reports consultancy fees from the European Respiratory Society, during the conduct of the study; consultancy fees from King's College Hospital NHS Foundation Trust, Academic Medical Research, AMC Medical Research BV, Asthma UK, Athens Medical School, Boehringer Ingelheim International GmbH, CHU de Toulouse, CIRO, DS Biologicals Ltd, École Polytechnique Fédérale de Lausanne, European Respiratory Society, FISEVI, Fluidic Analytics Ltd, Fraunhofer IGB, Fraunhofer ITEM, GSK R\&D Ltd, Holland and Knight, Karolinska Institutet Fakturor, KU Leuven, Longfonds, National Heart and Lung Institute, Novartis Pharma AG, Owlstone Medical Ltd, PExA AB, UCB Biopharma SPRL, UCB Biosciences GmbH, Umeå University, University Hospital Southampton NHS Foundation Trust, Università Campus Bio-Medico di Roma, Universita Cattolica Del Sacro 
Cuore, Universität Ulm, University of Bern, University of Edinburgh, University of Hull, University of Leicester, University of Loughborough, University of Luxembourg, University of Manchester, University of Nottingham, Vlaams Brabant, Dienst Europa, Imperial College London, Boehringer Ingelheim, Breathomix, Gossamer Bio, AstraZeneca and CIBER, outside the submitted work. R. Djukanovic reports receiving fees for lectures at symposia organised by Novartis, AstraZeneca and Teva, consultation for Teva and Novartis as member of advisory boards, and participation in a scientific discussion about asthma organised by GSK; and is a co-founder and current consultant, and has shares in Synairgen, a University of Southampton spin out company. A.H. Maitland-van der Zee reports personal fees for participating in an advisory board from AstraZeneca and Boehringer Ingelheim, unrestricted research grants from Boehringer Ingelheim and GSK.

\section{References}

1 Djukanovic R, Adcock IM, Anderson G, et al. The Severe Heterogeneous Asthma Research collaboration, Patient-centred (SHARP) ERS Clinical Research Collaboration: a new dawn in asthma research. Eur Respir J 2018; 52: 1801671.

2 Israel E, Reddel HK. Severe and difficult-to-treat asthma in adults. N Engl J Med 2017; 377: 965-976.

3 Chung KF, Wenzel SE, Brozek JL, et al. International ERS/ATS guidelines on definition, evaluation and treatment of severe asthma. Eur Respir J 2014; 43: 343-373.

4 Global Initiative for Asthma. Difficult-to-Treat \& Severe Asthma in Adolescent and Adult Patients. Diagnosis and Management. 2019. https://ginasthma.org/wp-content/uploads/2019/04/GINA-Severe-asthma-Pocket-Guidev2.0-wms-1.pdf Date last accessed: September 29, 2019.

5 Pavord ID, Beasley R, Agusti A, et al. After asthma: redefining airways diseases. Lancet 2018; 391: 350-400. Bush A, Pavord ID. After the asthmas: Star Wars and Star Trek. Eur Respir J 2017; 50: 1701362.

7 Royal College of Physicians. Why Asthma Still Kills: The National Review of Asthma Deaths (NRAD) Confidential Enquiry Report. London, RCP, 2014.

8 Amelink M, de Nijs SB, Berger M, et al. Non-atopic males with adult onset asthma are at risk of persistent airflow limitation. Clin Exp Allergy 2012; 42: 769-774.

9 Global Initiative for Asthma. Global Strategy for Asthma Management and Prevention. 2018. https://ginasthma. org/wp-content/uploads/2018/04/wms-GINA-2018-report-V1.3-002.pdf Date last accessed: September 29, 2019.

10 Pavord ID, Korn S, Howarth P, et al. Mepolizumab for severe eosinophilic asthma (DREAM): a multicentre, double-blind, placebo-controlled trial. Lancet 2012; 380: 651-659.

11 Bel EH, Wenzel SE, Thompson PJ, et al. Oral glucocorticoid-sparing effect of mepolizumab in eosinophilic asthma. N Engl J Med 2014; 371: 1189-1197.

12 National Institute for Health and Care Excellence. Reslizumab for treating severe eosinophilic asthma. Technical appraisal guidance 479. 2017. www.nice.org.uk/guidance/ta479/resources/reslizumab-for-treating-severeeosinophilic-asthma-pdf-82604974420933 Date last accessed: September 29, 2019.

13 National Institute for Health and Care Excellence. Omalizumab for treating severe persistent allergic asthma. Technology appraisal guidance 278. 2013. www.nice.org.uk/guidance/ta278/resources/omalizumab-for-treatingsevere-persistent-allergic-asthma-pdf-82600619176645 Date last accessed: September 29, 2019.

14 National Institute for Health and Care Excellence. Mepolizumab for treating severe refractory eosinophilic asthma. Technology appraisal guidance 431. 2017. www.nice.org.uk/guidance/ta431/resources/mepolizumab-for-treatingsevere-refractory-eosinophilic-asthma-pdf-82604719119301 Date last accessed: September 29, 2019.

15 Schleich F, Brusselle G, Louis R, et al. Heterogeneity of phenotypes in severe asthmatics. The Belgian Severe Asthma Registry (BSAR). Respir Med 2014; 108: 1723-1732.

16 Heaney LG, Brightling CE, Menzies-Gow A, et al. Refractory asthma in the UK: cross-sectional findings from a UK multicentre registry. Thorax 2010; 65: 787-794

17 Heffler E, Blasi F, Latorre M, et al. The Severe Asthma Network in Italy (SANI): findings and perspectives. J Allergy Clin Immunol Pract 2019; 7: 1462-1468.

18 de Vries R, Dagelet YWF, Spoor P, et al. Clinical and inflammatory phenotyping by breathomics in chronic airway diseases irrespective of the diagnostic label. Eur Respir J 2018; 51: 1701817.

19 Moore WC, Fitzpatrick AM, Li X, et al. Clinical heterogeneity in the severe asthma research program. Ann Am Thorac Soc 2013; 10: S118-S124.

20 Hekking PPW, Wener RR, Amelink M, et al. The prevalence of severe refractory asthma. J Allergy Clin Immunol 2015; 135: 896-902. 